\title{
Control Semigráfico de Análisis Elástico para ciertas Estructuras de Edificación
}

\author{
Agustin G. Lacort \\ Departamento de Arquitectura. ETSA, Universidad del País Vasco (UPV-EHU), \\ Plaza de Oñati, 2, 20018 San Sebastián-Donostia, España (e-mail: agustingregorio.lacort@ehu.es)
}

Recibido Jul. 31, 2014; Aceptado Oct. 20, 2014; Versión final recibida Ene. 7, 2014

\begin{abstract}
Resumen
El presente estudio es una modificación de un procedimiento de equilibrio manual descrito anteriormente por el autor que analizaba exactamente y en teoría de primer orden ciertos pórticos espaciales. Con la presente modificación el método se transforma en otro semigráfico más adecuado para emplearlo manualmente. Basado en dos grafos y en unas reglas de aplicación visual menores en número y complejidad que las del procedimiento original, el método se adapta también a otro de compatibilidad. Adicionalmente se aportan modelos adecuados para ser analizados mediante ambos procedimientos sin recurrir al álgebra matricial. El procedimiento expuesto sugiere una explicación de las operaciones realizadas con el método de Gauss de resolución de sistemas de ecuaciones basada en el comportamiento mecánico del modelo.
\end{abstract}

Palabras clave: análisis elástico, grafos, estructuras de edificación, métodos semigráficos

\section{Semigraphic Control of Elastic Analysis for Certain Building Structures}

\begin{abstract}
This study is a modification of a manual equilibrium procedure described previously by the author that analysed certain spatial porticoes exactly and in terms of first-order theory. With this modification the method becomes another semigraph more suitable to be manually used. Based on two graphs and a set of visual application rules that are fewer in number and less complex than in the original procedure, the method is also adapted itself to a compatibility system. Models are also provided that are suitable for analysis by both procedures without using matrix algebra. The procedure shown suggests an explanation of the operations performed with the Gauss method for resolving systems of equations based on the mechanical behaviour of the model.
\end{abstract}

Keywords: elastic analysis, graphs, building structures, semigraphic methods 


\section{INTRODUCCIÓN}

En los problemas de ingeniería mecánica debido a la mayor complejidad de los modelos y a la profundidad de los análisis es cada vez más importante desarrollar métodos informáticos que requieran menor tiempo de cálculo. Esta reducción de tiempo se consigue evolucionando los procedimientos analíticos en lugar de los conceptos mecánicos ya que éstos se consideran incuestionables. Actualmente los procedimientos analíticos se optimizan combinando una rama de la Matemática Aplicada, llamada Teoría de Grafos, con otras disciplinas como es la Ciencia Computacional. De esta forma se han desarrollado algoritmos que mejoran los análisis no lineales, dinámicos y de fluencia de grandes puentes (Keitel et al, 2011) y otros que optimizan el cálculo de los valores propios de las frecuencias naturales de vibración de ciertas estructuras (Kaveh, 2009). La aplicación de la Teoría de Grafos ha permitido también perfeccionar procedimientos que estudian el comportamiento de grandes pórticos bajo acciones estáticas; así, otros trabajos han conseguido mejorar los análisis de estabilidad reduciendo el tiempo de cálculo de los valores propios de las cargas de pandeo (Kaveh et al, 2006). Los análisis elásticos en Teoría de Primer orden se han optimizado al interactuar también la Teoría de Grafos con la de matrices dispersas (Spielman, 2010). Además existen líneas de investigación en métodos simplificados derivados de todos estos procedimientos. Según Dobry (2014) aquellos que simplifiquen el volumen de cálculo de los análisis avanzados sin perder demasiada precisión resultan especialmente interesantes para ser aplicados durante las primeras etapas del diseño ingenieril y son un buen complemento para el técnico proyectista. La mayor parte de estos procedimientos son de tipo numérico, como es el de Seung-Eok et al (2001), ideado para diseñar pórticos tridimensionales teniendo en cuenta los efectos P- $\delta$ y P- $\Delta$ derivados de las deformadas, o también el de Yanyan y Hong (2014), concebido para estudiar la respuesta estructural de ciertos embarcaderos frente a impactos de barcos.

Existen otros métodos simplificados que son de carácter gráfico, algunos de los cuales están concebidos para analizar estructuras de edificación en Teoría de Primer Orden. Cuando los modelos son hiperestáticos estos procedimientos contemplan hipótesis que evitan el efecto de las redundantes y la compatibilidad de deformaciones entre los elementos; y en algunos casos sirven para optimizar formas estructurales de obra nueva. Según Huanyun y Chunhui (2013) sus versiones manuales tienen un papel irremplazable en el desarrollo de las habilidades de los futuros técnicos. Adicionalmente se han publicado revisiones, como la de Fivet y Zastavni, (2012) o la de Huerta (2006), sobre métodos de diseño gráficos empleados en el pasado por algunos técnicos relevantes como Maillart y Gaudí, que aún se consideran interesantes. Estas versiones manuales se complementan con otras informáticas (Van Mele et al, 2012; Beghini et al, 2014) que, al adoptar los principios clave de la estática gráfica y diversas técnicas actuales de optimización, realizan más eficazmente lo que fue comprendido de forma manual. Existen además otros métodos gráficos que sirven para estudiar modelos hiperestáticos preexistentes, como son el de Nilesh et al (2012) que permite analizar la estabilidad de taludes, el de Block y Lachauer (2014) concebido para estudiar bóvedas antiguas de albañilería, o bien el de Sánchez-Beitia (2013) ideado para predecir el mecanismo de colapso de determinados arcos fisurados de piedra.

El trabajo que se expone podría formar parte de estos últimos procedimientos gráficos, ya que en él se sugiere una manera semigráfica de analizar determinados modelos de estructuras lineales hiperestáticas definidos previamente. Considerando el efecto que tienen sus redundantes, empleando ciertos grafos y en parte la Teoría que los gobierna, el nuevo procedimiento propone una manera de visualizar las operaciones realizadas por otro método numérico exacto descrito anteriormente (Lacort, 2011) que está fundamentado en procedimientos manuales clásicos de equilibrio como es el de Cross. Para ello utiliza dos grafos $\mathrm{G}_{1}$ y $\mathrm{G}_{2}$ que actúan como soporte donde se realizan las operaciones del método, empleando unas reglas visuales menores en número y complejidad que las del procedimiento original. Mediante $\mathrm{G}_{2}$ se calcula la deformada $y$, a partir de esta, con $\mathrm{G}_{1}$ se obtienen los esfuerzos en las barras. Complementariamente, el presente método se adapta a otro de compatibilidad para determinar semigráficamente los flectores en vigas continuas realizando menos operaciones. La exposición comienza describiendo y justificando la obtención del grafo $G_{2}$ de un modelo indesplazable partiendo del grafo $G_{1}$ y la manera de calcular su deformada. Posteriormente se muestran unas tipologías de mallas adecuadas y la manera semigráfica de analizarlas. Finalmente la exposición termina con un ejemplo.

\section{METODOLOGÍA}

El procedimiento original analizaba figuras espaciales bajo acciones nodales descompuestas en estados de carga que se denominaron "estados primarios" porque cada uno producía una deformada inmediata de calcular. En cada estado primario existía una acción "activa" que producía el movimiento de algunos nudos así como varias acciones "restrictivas" que impedían el resto de los movimientos nodales. En el presente trabajo cada estado de carga se ha denominado $\varepsilon$ "IJk.... El subíndice I es el movimiento del nudo donde actúa la acción activa y los subíndices $\mathrm{J}, \mathrm{K}, \ldots$ los restantes movimientos nodales. El orden de estos 
subíndices indica una relación entre ellos, de modo que cada uno depende del que está situado a su izquierda. Un estado se ha denominado "inicial" si su deformada está en función de un único movimiento; "intermedio" si depende de varios y "último" si depende de los que gobiernan la deformada total. Cada $\varepsilon$ "IJk...

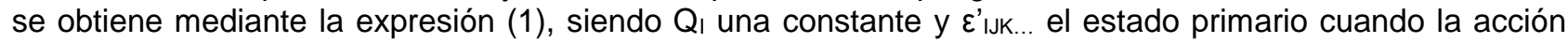
activa es unitaria, que a su vez se calculará combinando estados primarios designados en general como $\varepsilon_{I J K \ldots}$

$$
\varepsilon^{\prime \prime}{ }_{\text {IJK .. }}=Q_{1} \cdot \varepsilon^{\prime}{ }_{\text {IJK .. }}
$$

La metodología se desarrolla en tres etapas. En las dos primeras se elaboran los grafos $G_{1}$ y $G_{2}$ que controlarán semigráficamente el análisis realizado en la última etapa. A continuación considerando las hipótesis manuales de deformación en vigas se describe cualitativamente el grafo $G_{1}$ del modelo de la Figura 1a y después se describe la obtención tanto del grafo $\mathrm{G}_{2}$ como del procedimiento para calcular con él una deformada.

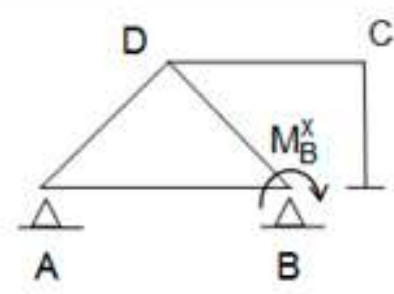

a)

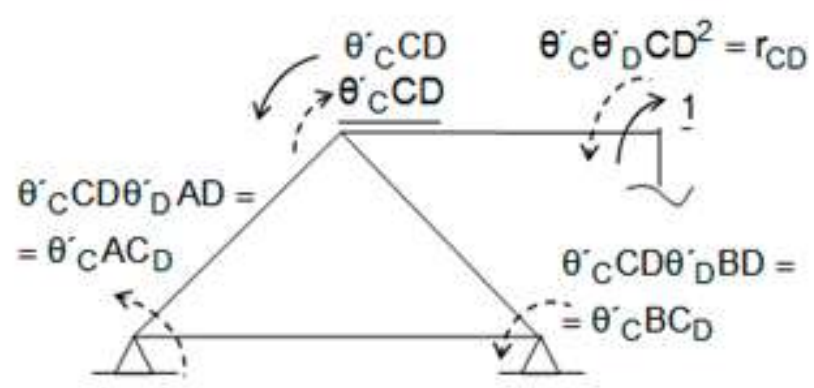

c)

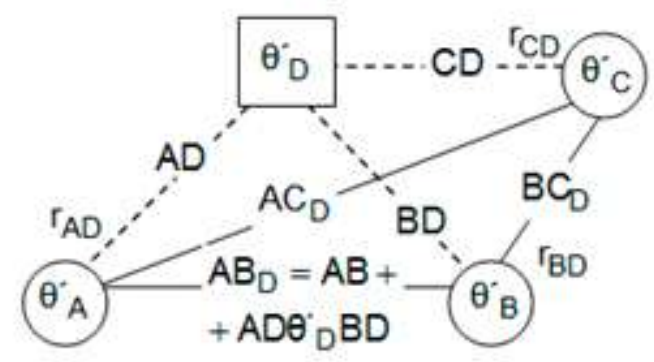

e)

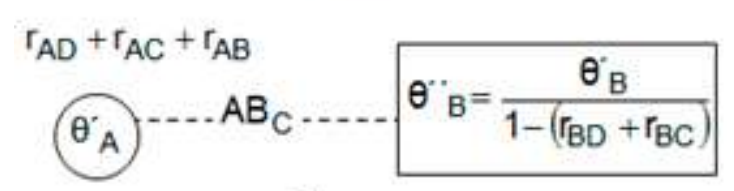

g)

$$
\theta^{*}{ }_{A}=\frac{\theta_{A}}{1-\Sigma r_{A}}
$$

h)

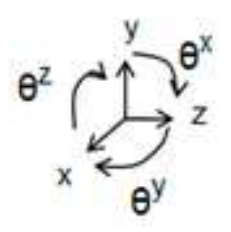

)$$
\text { . }
$$ 


\section{Descripción del grafo $G_{1}$}

En la etapa 1 se ha elaborado el grafo $G_{1}=\left(V^{\prime}, E^{\prime}\right)$ que aparece en Fig.1b considerando la geometría del modelo y las propiedades del material. Los valores de sus vértices $V^{\prime}$ y arcos $E^{\prime}$ dependen de los estados iniciales unitarios aplicables en la Figura 1a. Por ejemplo, el valor de $V^{\prime} \mathrm{c}$ es el giro $\theta^{\prime} \mathrm{c}$ determinado por la deformada del estado $\varepsilon^{\prime} c$, cuyas acciones se muestran subrayadas en Fig.1c. Por otra parte el valor del arco que une $V^{\prime} C$ con el vértice adyacente $V^{\prime}$ ' es el factor $C D$ de la barra correspondiente que, multiplicado por $\theta^{\prime} \mathrm{c}$ determina el momento restrictivo de $\varepsilon^{\prime} \mathrm{c}$ en $\mathrm{D}$, cambiado de signo.

\section{Elaboración del grafo $G_{2}$}

Combinando los estados iniciales que componen $\mathrm{G}_{1}$, en la etapa 2 se obtienen los estados unitarios $\varepsilon^{\prime}$ IJk... que se emplearán para descomponer el estado de cargas de la estructura. Sus deformadas están solapadas y a la vez concatenadas entre sí, existiendo múltiples soluciones de estados $\varepsilon^{\prime}$ IJk.... Para descomponer el momento $M^{x_{B}}$ del ejemplo se ha considerado el estado $\varepsilon^{\prime}$, los intermedios $\varepsilon^{\prime}{ }_{C D}$ y $\varepsilon^{\prime}{ }_{B C D}$, y el estado último $\varepsilon^{\prime}$ ABCD. Todos ellos se obtendrán progresivamente combinando otros estados de carga que abarcan cada vez mayor número de movimientos nodales. Se comienza calculando unos estados intermedios $\varepsilon_{A D}, \varepsilon_{B D}$ y $\varepsilon_{C D}$, cada uno obtenido sumando dos estados iniciales. En Fig.1c se ha determinado cualitativamente $\varepsilon_{C D}$ añadiendo a $\varepsilon^{\prime} c$ otro estado $\varepsilon_{D}$ que deja girar a $D$ con libertad y cuyo momento restrictivo en $\mathrm{C}$ menor que la unidad se ha denominado $\mathrm{r}_{\mathrm{CD}}$. Estas operaciones y otras similares con las que se obtienen $\varepsilon_{A D}$ y $\varepsilon_{B D}$ transforman el modelo original en el de Fig.1d a partir del cual se obtendrá $\varepsilon^{\prime} C D$ eliminando $r_{C D}$. Esto se consigue añadiendo estados intermedios proporcionales a $\varepsilon_{C D}$ de manera que el momento activo de cada estado aportado anule en $\mathrm{C}$ el restrictivo del último estado aplicado. Así se obtiene en $\mathrm{C}$ un momento exterior unitario y un momento activo de valor $1 /\left(1-\mathrm{r}_{\mathrm{CD}}\right)$ que multiplicado por $\theta^{\prime} \mathrm{c}$ determina la rotación $\theta^{\prime \prime} \mathrm{C}$ debido a $\varepsilon^{\prime} \mathrm{CD}$, considerando la influencia de D. Partiendo del modelo de Fig.1d y combinando $\varepsilon_{A D}$ y $\varepsilon_{B D}$ con $\varepsilon^{\prime} C D$ se obtienen otros estados intermedios $\varepsilon_{A C D}$ y $\varepsilon_{B C D}$ con los que se determina $\varepsilon^{\prime} \mathrm{BCD}$ eliminando del mismo modo el restrictivo en $\mathrm{B}$. Posteriormente, repitiendo el procedimiento se obtiene $\varepsilon^{\prime} A B C D$. Finalmente a partir de los resultados obtenidos se determina el grafo $G_{2}$ de Fig. 1 i.

Se ha advertido que establecer una dependencia entre el giro de un nudo $\mathrm{N}$ y los giros de sus nudos adyacentes equivale a transformar $\mathrm{G}_{1}$ en otro grafo $\sin \mathrm{V}_{\mathrm{N}}$ realizando dos operaciones. La primera modifica el movimiento de $\mathrm{N}$ debido a la influencia $\Sigma \mathrm{r}_{\mathrm{NM}}$ que tienen sobre él los movimientos de otros nudos $\mathrm{M}$ adyacentes previamente eliminados. Esta modificación se obtiene aplicando (2), siendo $\theta^{\prime \prime} \mathrm{N}$ el valor del vértice $V^{\prime \prime} N$ del grafo $G_{2}$. La segunda operación modifica $G_{1}$ sin $N$ debido a la influencia que tiene el movimiento de este nudo sobre el resto del grafo. Se obtiene transformando el arco IJ existente entre cada par de vértices $V^{\prime}$ 'ı, $V^{\prime} J$ adyacentes a $V^{\prime} N$ según (3) y además disponiendo unos coeficientes riN de valor (4) cerca de cada vértice $V_{I}$ adyacente a $V_{N}$. Estas operaciones aplicadas repetidamente en $G_{1}$ (Figs.1e,f,g,h) reducen el tamaño del grafo hasta hacerlo desaparecer, y con los vértices y arcos obtenidos se compone el grafo $G_{2}=\left(V^{\prime \prime} 1, E^{\prime \prime}\right)$ de Fig.1i. Sus vértices $V^{\prime \prime}$ quedan ordenados en niveles según el orden de eliminación de los vértices $V^{\prime}$.

$$
\begin{aligned}
& \theta^{\prime \prime}{ }_{N}=\frac{\theta^{\prime}}{1-\Sigma r_{N M}} \\
& I_{N}=I J+I N \theta^{\prime \prime}{ }_{N} J N \\
& r_{I N}=\theta^{\prime} \theta^{\prime \prime}{ }_{N} I^{2}
\end{aligned}
$$

\section{Obtención de la deformada}

La deformada se obtiene sumando las de los estados $\varepsilon$ "IJk... que se han determinado calculando sus coeficientes $Q_{1}$ con $\mathrm{G}_{2}$. Uno de estos coeficientes vale la acción exterior asociada al primer vértice $\mathrm{V}^{\prime}$ eliminado que esté cargado externamente. Los restantes se obtienen en $G_{2}$ a partir del coeficiente $Q_{1}$

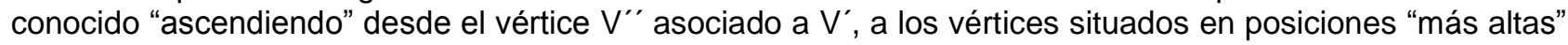
mediante los arcos $\mathrm{E}^{\prime \prime}$. Así se han obtenido los coeficientes (5) y (6) considerando $\mathrm{G}_{2}$ de Fig.1i. La suma de las deformadas de los estados $\varepsilon$ "IJk... se obtiene también con $\mathrm{G}_{2}$ "descendiendo" desde el vértice $\mathrm{V}^{\text {" situado }}$ en el nivel "más alto" hasta llegar al situado en el nivel "más bajo" y sumando los movimientos parciales obtenidos en cada vértice. El proceso completo se automatiza en Fig.1i: determinado primeramente el giro parcial $\theta_{B 1}$, se "asciende" por $A B_{C}$ y se obtiene la rotación total en $A$. Desde aquí "descendiendo" por $A D$, $A C_{D}$ y $A B_{C}$ se calculan nuevos giros parciales y $\theta_{B}$. A continuación "descendiendo" desde $B$ se calcula 
primero $\theta_{\mathrm{c}}$ y por último $\theta_{\mathrm{D}}$. La deformada también se ha obtenido con el grafo de Fig.2a realizando menos operaciones.

$\mathrm{Q}_{\mathrm{B}}=\mathrm{M}_{\mathrm{B}}$

$Q_{A}=Q_{B} A B_{C}$

\section{Algunas observaciones}

Se ha apreciado que $\mathrm{G}_{1}$ coincide con el grafo de eliminación derivado de la matriz dispersa procedente del sistema de ecuaciones de equilibrio de los nudos, y que existe una analogía entre la segunda operación descrita más arriba y el Teorema I de Parter (1962) empleado para aplicar simbólicamente el procedimiento de Gauss sobre este grafo de eliminación. Ello sugiere que el procedimiento iterativo expuesto aporta una explicación cualitativa de las operaciones realizadas por otro directo preexistente basada en el comportamiento mecánico del modelo. Por otra parte se advierte que la complejidad formal de $\mathrm{G}_{2}$ y el volumen de operaciones para su determinación dependen de los estados $\varepsilon^{\prime}$ considerados en la etapa 2, y por tanto del orden de eliminación de los vértices $V^{\prime}$. Para reducir esta complejidad se podría eliminar los vértices de $\mathrm{G}_{1}$ comenzando por aquellos que tuvieran menor grado, lo que equivaldría a aplicar el algoritmo de grado mínimo, aunque no siempre ello conduzca a una solución óptima (De la Fuente, 1996). Sin embargo es posible aplicar eficazmente este procedimiento sobre los grafos $\mathrm{G}_{1}$ de determinados pórticos, como son los de Figs.3a,b,c si se comienza eliminando los vértices relacionados con las rotaciones de sus nudos. En Fig.2a se muestra un grafo $\mathrm{G}_{2}$ alternativo al de Fig.1i que resulta de haber eliminado inicialmente V'A y V'c. Otras estrategias recogidas en la literatura ya clásica para resolver óptimamente sistemas de ecuaciones de matriz hueca (Pissanetzky, 1984) no han sido consideradas para obtener los grafos $\mathrm{G}_{2}$ porque podrían resultar demasiado laboriosas. Adicionalmente se ha observado que un grafo $\mathrm{G}_{3}$ resultante de modificar $G_{1}$ con (3) y (4) contemplando los valores iniciales de $V^{\prime}$ y obteniendo después con (2) los valores de los vértices V' es parecido al $G_{2}$ correspondiente y más sencillo de calcular. Por ello se sugiere $\mathrm{G}_{3}$ para realizar análisis aproximados ya que sus resultados no parecen desviarse más de un $10 \%$ respecto de los valores exactos.

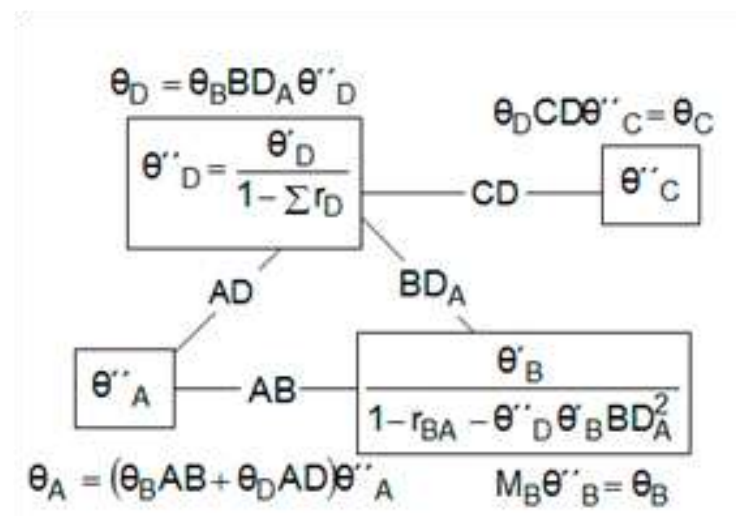

a)

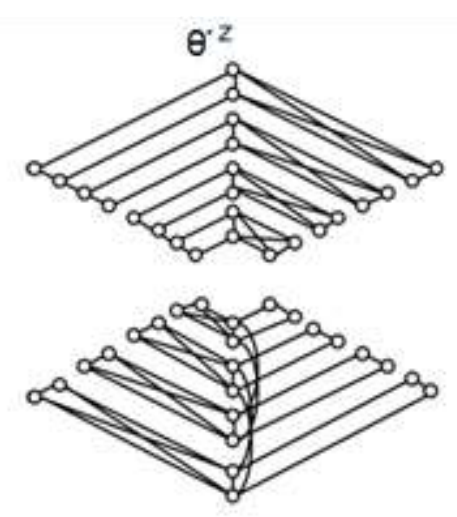

b)

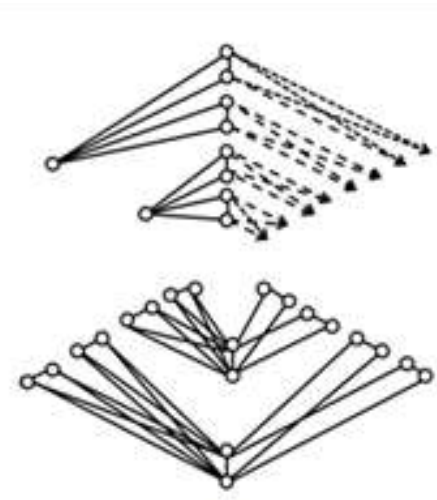

c)

Fig.2: Ejemplos de grafos. a) $\mathrm{G}_{2}$ alternativo al de Fig.1i y deformada resultante; b), c): $\mathrm{G}_{1}$ asociados a Fig.3a sin considerar y contemplando las hipótesis manuales, respectivamente

\section{APLICACIÓN EN ESTRUCTURAS DE EDIFICACIÓN}

Considerando las hipótesis manuales se han detectado tres tipologías de mallas espaciales de un piso aceptables de ser analizadas con este procedimiento, dos de las cuales podrían ser válidas para cubrir grandes superficies. La primera la constituyen combinaciones de pérgolas de directriz recta formadas con nudos rígidos (Fig.3a); sus grafos $\mathrm{G}_{1}$, simplificados al considerar inextensibles los tramos, tienen formas sencillas (Fig.3I). Si los nudos internos fueran articulados, estos grafos no simplificados serían igualmente de poca complejidad. La segunda tipología está formada con pórticos de un vano paralelos o perpendiculares entre sí y arriostrados con barras articuladas (Fig.3b). La organización de estos pórticos permitiría disponer de claraboyas que iluminaran cenitalmente el interior. Sus grafos $\mathrm{G}_{1}$ simplificados (Fig.2c) son también sencillos e inconexos. En caso de considerar un único modelo de pilar y de viga, el cálculo de $\mathrm{G}_{2}$ requeriría realizar muy pocas operaciones. También es aceptable el $\mathrm{G}_{1}$ resultante de considerar el alargamiento de los tramos (Fig.2b). La tercera tipología está formada con pórticos $\pi$, paralelos entre sí y arriostrados transversalmente (Fig.3c). Sus grafos $\mathrm{G}_{1}$ simplificados son más complejos que los de las anteriores tipologías, pero se pueden sustituir por otros relativamente sencillos sin cometer 
errores importantes. Esta sustitución se puede realizar cuando las fuerzas están orientadas respecto de $\pi$ como se muestra en la Figura $3 c$ y si se considera, como sugiere Calavera (2008), que la deformada de $\pi$ por cada fuerza dependa únicamente del movimiento del nudo donde esté aplicada y de los movimientos de los nudos de $\pi$ contiguos. La simplificación también se ha considerado razonable cuando existan momentos en $\pi$ orientados como los de la Figura 3c. A continuación se aportan unas reglas nemotécnicas para analizar por equilibrio y semigráficamente estas tres tipologías. Unas reglas definen el $\mathrm{G}_{1}$ de una malla ordinaria genérica partiendo de la geometría del modelo; otras lo simplifican contemplando inextensibles los tramos y unas terceras calculan los esfuerzos de las barras partiendo de $G_{1}$ sin simplificar y de la deformada. Finalmente se adapta el procedimiento a otro de compatibilidad para calcular más eficazmente los diagramas de flexión producidos en vigas continuas.

\section{Reglas para obtener $G_{1}$ cuando el análisis es de equilibrio}

Nombrando de manera consecutiva los nudos internos $\mathrm{n}$ de la malla, se comienza a dibujar $\mathrm{G}_{1}$ disponiendo sus $V^{\prime}$ de modo que los que representan los movimientos incógnita de cada $n$ formen las esquinas de un hexágono regular. Cada V' se representa con un círculo en cuyo interior se dispondrá su valor v' que es un movimiento $\theta^{\prime}$ o $\delta^{\prime}$ similar a los de Fig.1b. Los vértices de cada hexágono se distribuyen como en Fig.3d y los hexágonos se disponen ordenada y concéntricamente respecto de un centro $\mathrm{K}$, alejándose de él y comenzando con el del nudo $\mathrm{A}$. De esta manera los vértices alineados con $\mathrm{K}$ tienen movimientos incógnita paralelos entre sí. Los hexágonos vinculados a los apoyos exteriores no se dibujan al tener movimientos nulos, tan sólo se representarán los arcos de las barras que los unen a $\mathrm{G}_{1}$.

A continuación se dibujan los arcos que están orientados respecto de $\mathrm{K}$ radial, diagonal o concéntricamente. Cada barra b puede contribuir con catorce arcos como máximo. Para situar en $\mathrm{G}_{1}$ los arcos de una barra unida a dos nudos designados I, J, se sugiere orientar previamente $G_{1}$ de manera que los vértices giro, cuyos superíndices coincidan con la orientación de b en el espacio, se sitúen en la parte superior del grafo. Así, los hexágonos de Fig.3d están orientados correctamente cuando se desea dibujar los arcos de una barra cuya directriz es paralela a OZ. Conforme a esta disposición se dibujan los arcos de b (Fig.3e) cuyos valores se recogen en unos coeficientes a que dependen de la orientación de la barra y de sus enlaces. La Tabla 1 describe los coeficientes a cuando la barra es biempotrada; en el caso de ser biarticulada, todos los valores son nulos salvo los de la columna $a_{2}$.

Tabla 1: Coeficientes $\alpha$ de una barra según su orientación en el espacio

\begin{tabular}{|c|c|c|c|c|c|c|c|c|}
\hline $\begin{array}{c}\text { Barra } \\
\text { paralela }\end{array}$ & $a_{1}$ & $a_{2}$ & $b_{1}$ & $b_{2}$ & $b_{3}$ & $c_{1}$ & $\mathrm{C}_{2}$ & $\mathrm{C}_{3}$ \\
\hline a OX & $\mathrm{GI}_{\mathrm{t}} / \mathrm{L}$ & $E A / L$ & $2 \mathrm{EI}_{\mathrm{z}} / \mathrm{L}$ & $3 b_{1} / L$ & $2 b_{2} / L$ & $2 \mathrm{EI}_{\mathrm{y}} / \mathrm{L}$ & $3 c_{1} / L$ & $2 c_{2} / L$ \\
\hline a OY & " & " & $2 \mathrm{EI} \mathrm{x}_{\mathrm{x}} / \mathrm{L}$ & “ & “ & $2 \mathrm{EI} \mathrm{I}_{\mathrm{Z}} / \mathrm{L}$ & “ & “ \\
\hline a OZ & " & " & $2 \mathrm{EI}_{\mathrm{y}} / \mathrm{L}$ & “ & " & $2 \mathrm{EI} \mathrm{I}_{\mathrm{x}} / \mathrm{L}$ & “ & " \\
\hline
\end{tabular}

Los signos de los arcos radiales siempre son los que aparecen en Fig.3e, y los de los arcos concéntricos y diagonales dependen de la posición en el espacio de los extremos I y $\mathrm{J}$ respecto del sistema de referencia global (Fig.1a). Según este sistema, cuando el sentido de I hacia $\mathrm{J}$ a lo largo de b es positivo, el signo de estos arcos es el que aparece en Fig.3e; cuando es negativo, el signo es el contrario. De este modo se obtiene un $G_{1}$ cuya forma general sugiere en muchos casos la imagen de una tela de araña. La Figura $3 j$ muestra el grafo $G_{1}$ sin completar que está vinculado a la pérgola de Fig.3a. Sus líneas continuas son los arcos de las barras orientadas horizontalmente; sus líneas discontinuas son los del pilar de esquina y los arcos concéntricos de las barras restantes. En Fig.3k se expone un detalle de este grafo y en Fig.2b y Fig.5a se muestran los $\mathrm{G}_{1}$ de Fig.3b y Fig.3c, respectivamente. Dispuestos los arcos se calcula el valor $\mathrm{v}^{\prime}$ de cada $V^{\prime}$ aplicando (7) y se sitúa en el círculo correspondiente. Los coeficientes a que intervienen son los de los arcos radiales convergentes en $V^{\prime}$ y valen doble cuando son $b_{1} \circ c_{1}$.

$v^{\prime}=\frac{1}{\Sigma|\alpha|}$

\section{Reglas para simplificar $G_{1}$ según hipótesis manuales}

$\mathrm{Si}$ se consideran inextensibles las barras, $\mathrm{G}_{1}$ se transforma en un dígrafo porque los vértices $\delta^{\prime y}$ desaparecen. Además se igualan los movimientos de los vértices $\delta^{\prime x} 0 \delta^{\prime z}$ que estén conectados entre sí mediante arcos de coeficientes a2. Como consecuencia, estos vértices denominados en general como $\delta^{\prime}$ se 
sustituyen por otro cuyo valor $\Delta^{\prime}$ se obtiene mediante (8). De esta forma el grafo teórico de Fig.3g se ha convertido en el de Fig.3h. Si además los vértices de la fila inferior de dicho grafo son también del tipo $\delta^{\prime}$ y están unidos mediante otros arcos $\mathrm{a}_{2}$, el grafo se transforma en el de Fig.3i. Procediendo de esta manera los grafos de Fig.2b y Fig.3j se convierten en los de Fig.2c y Fig.3l respectivamente. En ambos casos las líneas continuas y vértices que conectan forman unos subgrafos con los que se obtiene $\mathrm{G}_{2}$ de manera sencilla. Se observa que si se suprimiera la rigidez torsional de los tramos al considerar su efecto despreciable, el cálculo de $\mathrm{G}_{2}$ no se simplificaría significativamente.

$$
\Delta_{1}^{\prime}=\frac{1}{\sum \frac{1}{\delta_{1}^{\prime}}-2 \sum \mathrm{a}_{2}}
$$

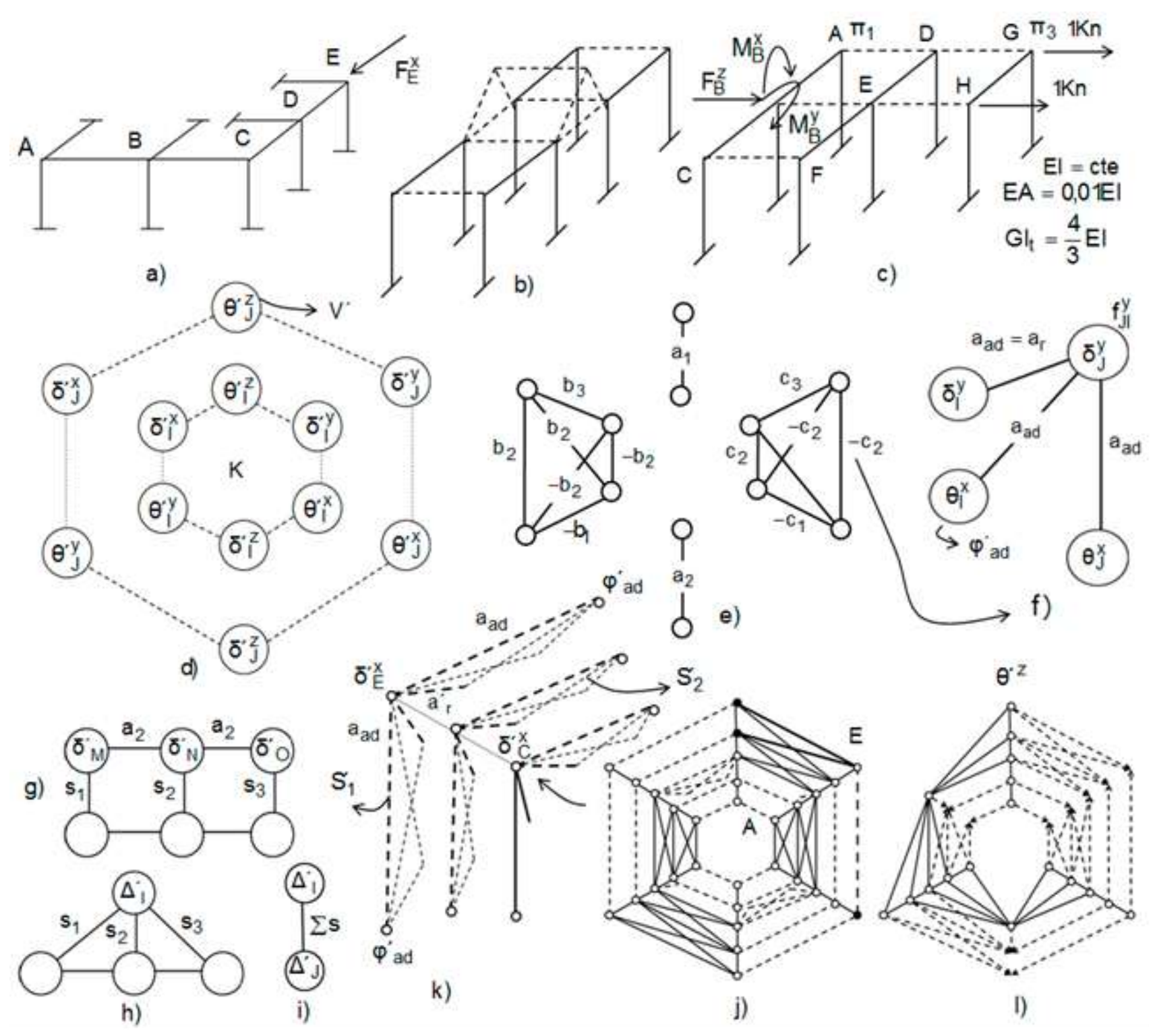

Fig.3: Tipologías y grafos. a), b),c) Tipologías (barra biempotrada: continua; articulada: discontinua); d) V' de dos nudos I, J consecutivos; e) Arcos de una barra; f) Subgrafo para calcular un cortante; g) G 1 teórico; h), i) Simplificaciones de $G_{1}$; j) $G_{1}$ de Fig.3a; k) Detalle; I) $G_{1}$ simplificado.

\section{Reglas para obtener semigráficamente los esfuerzos de una barra}

La solicitación en una dirección $\mathrm{k}$ del espacio situada en el extremo I de una barra b se puede obtener

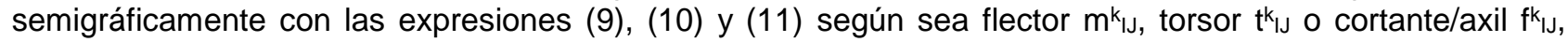
respectivamente, considerando los valores de los arcos de un subgrafo $S$ de $G_{1}$ y los movimientos de la deformada asociados a sus vértices. Estos vértices son: i) el vértice $\theta^{\prime \prime} k_{1}$ cuando la solicitación es un momento, o bien el vértice $\delta^{\prime} k_{\text {I }}$ cuando la solicitación es una fuerza; ii) los vértices $\varphi^{\prime}$ ad adyacentes al i) y que 
estén unidos a él mediante arcos de la barra b. Los valores de estos arcos se designan en general con aad, de los cuales el arco radial también se designa con $a_{r}$. A modo de ejemplo en Fig.3f se muestra el subgrafo que determina el cortante paralelo a OY en el extremo $\mathrm{J}$ de la barra de Fig.3e. La numeración de los extremos I y $\mathrm{J}$ de la barra no afecta al resultado del cálculo y el criterio de signos es el de Fig.1a.

$$
\begin{aligned}
& \mathrm{m}_{\mathrm{IJ}}^{\mathrm{k}}=-2 \mathrm{a}_{\mathrm{r}} \theta_{1}^{\mathrm{k}}-\sum \mathrm{a}_{\mathrm{ad}} \varphi_{\mathrm{ad}} \\
& \mathrm{t}_{\mathrm{IJ}}^{\mathrm{k}}=\mathrm{a}_{\mathrm{r}} \theta_{l}^{\mathrm{k}}-\sum \mathrm{a}_{\mathrm{ad}} \varphi_{\mathrm{ad}} \\
& \mathrm{f}_{\mathrm{IJ}}^{\mathrm{k}}=\mathrm{a}_{\mathrm{r}} \delta_{l}^{\mathrm{k}}-\sum \mathrm{a}_{\mathrm{ad}} \varphi_{\mathrm{ad}} \\
& \mathrm{f}_{\mathrm{IJ}}^{\mathrm{k}}=\mathrm{F}_{1}^{\mathrm{k}}-\Delta_{l}^{\mathrm{k}} \sum \mathrm{a}_{\mathrm{r}}+\sum \mathrm{a}_{\mathrm{ad}} \varphi_{\mathrm{ad}}
\end{aligned}
$$

Por otra parte, las solicitaciones de una barra $b$ inextensible se pueden determinar con subgrafos de $G_{1}$ no simplificados y con la deformada determinada previamente con el $\mathrm{G}_{1}$ simplificado. Mientras los momentos y cortantes se obtienen como en el caso anterior, el axil se determina mediante (12) empleando otro subgrafo $\mathrm{S}^{\prime}$, cuyos arcos provienen de b y de algunas barras indesplazables unidas a ella. Cuando existe una sola barra IJ desplazable en una dirección $k$, los vértices de $S^{\prime}$ son: i) el vértice $\delta^{\prime} k_{1}$ cuyo movimiento vale $\Delta^{k_{1}}$; ii) los vértices $\varphi^{\prime}$ ad adyacentes a $\delta^{\prime} k_{I}$ (salvo $\delta^{\prime} k_{\jmath}$ ) y unidos a él mediante arcos cuyos valores se designan en (12) con $\mathrm{a}_{\mathrm{ad}}$, de los cuales los radiales también se designan con $\mathrm{a}_{\mathrm{r}}$. El término $\mathrm{F}^{\mathrm{k}}$ l es una posible fuerza exterior existente en I y $\varphi_{\text {ad }}$ son los movimientos de $\varphi^{\prime}$ ad. Si existen varios tramos desplazables alineados, sus axiles se pueden calcular consecutivamente a partir del axil de una de las barras laterales. Por ejemplo $f^{\prime} x_{E D}$ de Fig.3a se puede obtener mediante (12) considerando los movimientos de los vértices $\delta^{\prime} x_{E}$ y $\varphi^{\prime}$ ad del subgrafo regruesado $S^{\prime}{ }_{1}$ de Fig.3k. Después se obtiene $f^{\prime} x_{C D}$ contemplando los movimientos y arcos de $S^{\prime}{ }_{2}$ regruesado, considerando que el primer término de (12) es $f^{\prime} x_{E D}+F_{D}$, si existiera una fuerza exterior en $D$. Si la barra no es desplazable el axil se obtiene igualmente con (12) prescindiendo del segundo término. En Fig.3j se muestra regruesado el subgrafo empleado para determinar el axil de un pilar de esquina.

\section{Adaptación del procedimiento a otro de compatibilidad}

El procedimiento adaptado a otro de compatibilidad puede ser adecuado para analizar manualmente modelos de bajo grado de hiperestaticidad estático o bien para analizar vigas continuas como la de la Figura $4 \mathrm{~b}$ considerando sus redundantes, al tener sus grafos $\mathrm{G}_{1}$ forma de rama. En este caso el procedimiento calcula el diagrama de momentos de las redundantes de las vigas partiendo de los giros relativos $\Delta \theta$ producidos en las rótulas y derivados de las cargas exteriores. El diagrama se obtiene descompuesto en otros diagramas parciales inmediatos de calcular. Cada diagrama parcial abarca diferente número de vanos y depende de una redundante expresada como XıJk. Mientras I es el nudo donde está aplicada esta redundante, los subíndices $\mathrm{J}, \mathrm{K}, \ldots$ son los nudos donde actúan otras que determinan el diagrama parcial. Existe una dependencia entre estas redundantes similar a la que tienen los movimientos de los nudos de un estado primario intermedio según el procedimiento de equilibrio.

a)

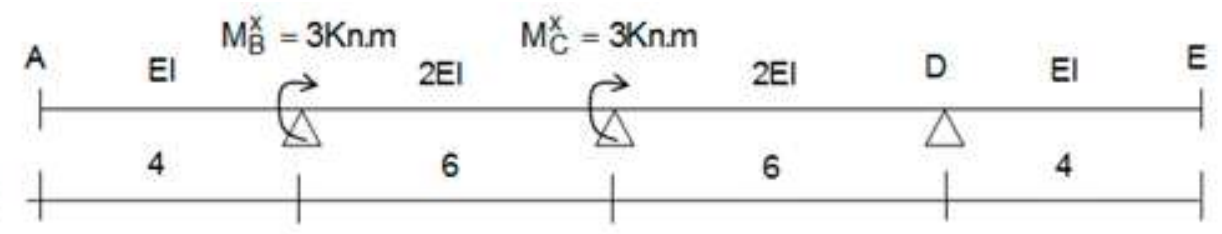

b)

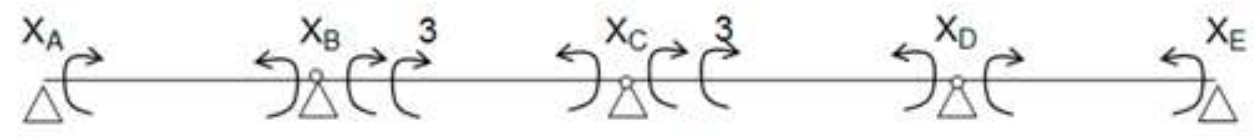

c)

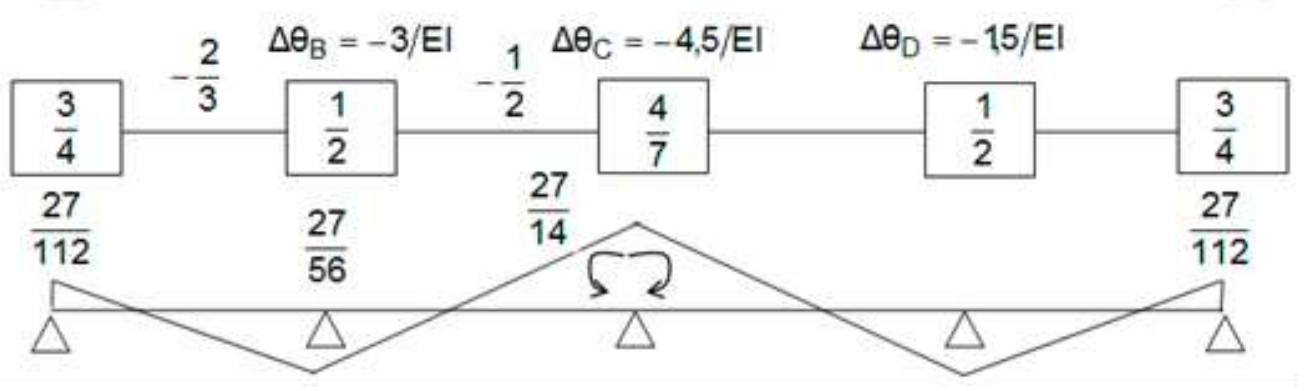

Fig.4: Análisis de una viga: a) Modelo; b) Isostática; c) Grafo $\mathrm{G}_{2}$; d) Flectores por la redundante en C 
A continuación se describe cualitativamente la obtención del diagrama parcial de momentos producido por la redundante $X_{B A}$ de Fig.4b suponiendo en $B$ un giro relativo (13) unitario. Dicho procedimiento es similar al expuesto más arriba para determinar $\varepsilon^{\prime} \mathrm{CD}$ : se comienza eliminando el giro relativo unitario en $B$ aplicando una redundante $X^{\prime}{ }_{B}$ que se obtiene con la ecuación de compatibilidad (14). Ello produce en $A$ otro giro relativo que vale el producto de $\mathrm{X}^{\prime}{ }_{\mathrm{B}}$ por (15) y se elimina añadiendo en $\mathrm{A}$ otra redundante que genera en $\mathrm{B}$ un nuevo giro relativo menor que la unidad, análogo a $r_{C D}$ de Fig.1c. Repitiendo el proceso ininterrumpidamente se consigue igualar completamente los movimientos de la isostática con los de la hiperestática en estos dos nudos, y sumando todas las redundantes parciales en A y B se obtienen los valores exactos de las redundantes que determinan el diagrama parcial.

$$
\begin{aligned}
& \Delta \theta_{B}=\theta_{B}^{i}(\curvearrowright)+\theta_{B}^{d}(\curvearrowleft) \\
& X_{B}^{\prime}=\frac{3}{\frac{L_{A B}}{E I_{A B}}+\frac{L_{B C}}{E I_{B C}}} \\
& A B=-\frac{L_{A B}}{6 E I_{A B}}
\end{aligned}
$$

\section{EJEMPLOS DE ANÁLISIS}

\section{Ejemplo 1: análisis de un modelo por compatibilidad}

De acuerdo con este planteamiento se ha analizado la viga de Fig.4a. Su grafo $G_{1}$ se ha obtenido considerando el sistema de referencia de Fig.1a. Mientras el valor de cada vértice $\mathrm{V}^{\prime}$ se ha calculado con (14) y equivale a la mitad de (7), el valor a de cada arco se ha calculado con (15) y equivale a $-1 / c_{2}$ obtenido con la Tabla 1. En Fig.4c se muestra $G_{2}$. Los valores de los vértices expresados en función de El se han obtenido eliminando simultáneamente $V^{\prime}{ }_{A}$ y $V^{\prime} E$, posteriormente $V_{B}^{\prime}$ y $V^{\prime}{ }_{D}$, y finalmente $V^{\prime}$ C. También se muestran los giros $\Delta \theta$ de la isostática debidos a $\mathrm{M}^{\mathrm{x}_{\mathrm{B}}}$ y $\mathrm{M}^{\mathrm{x}_{C}}$ a partir de los cuales se han calculado los diagramas de flectores de cada redundante, como es el diagrama de la redundante en $\mathrm{C}$ que aparece en Fig.4d.

\section{Ejemplo 2: análisis de un modelo por equilibrio}

Considerando las hipótesis del cálculo manual se desea determinar semigráficamente el axil de la barra BE y la deformada del pórtico $\pi_{1}$ de la malla de la Figura $3 c$ compuesta con barras de sección cuadrada y de 4 metros de longitud cuando está sometida a dos acciones horizontales de $1 \mathrm{Kn}$ en $\mathrm{G}$ y H. El grafo $\mathrm{G}_{1}$ no simplificado se define a partir de Fig.3c y de los coeficientes a de la Tabla 1 expresados en función de EI aplicando las reglas descritas más arriba. El resultado es un grafo inconexo formado por cuatro subgrafos $S$ que en Fig.5a aparecen sin completar al faltarles los arcos radiales y diagonales de los pilares. El subgrafo $\mathrm{S}_{4}$ está formado por tres subgrafos $\mathrm{S}^{\prime}$ conectados entre sí mediante unos arcos $\mathrm{a}_{2}$ procedentes de los arriostramientos. Estos arcos y los vértices que unen forman otro subgrafo $\mathrm{S}_{4}^{\prime}$ (Fig.5b) que se convierte en $\mathrm{S}_{4 S}^{\prime}$ (Fig.5d) al aplicar (8) si se consideran inextensibles los tramos. Así $\mathrm{S}_{4}$ se transforma en una figura espacial que tiene un eje central, cuyos vértices, $\Delta^{\prime} z_{A}, \Delta^{\prime} z_{B}, y \Delta^{\prime} z_{C}$ expresados en función de 1/El valen 8/9, $2 / 3$ y $4 / 3$ respectivamente.

La forma general de esta figura también se puede determinar realizando menos operaciones partiendo de la geometría del modelo y aplicando unas reglas nemotécnicas específicas. Sin embargo se prefiere obtenerla a partir del grafo $G_{1}$ sin simplificar al ser empleable este procedimiento a cualquier tipología. Aplicando (2), (3) y (4) en el subgrafo de Fig.5d se obtiene un grafo G2 que permite calcular la deformada. La obtención de este grafo se consigue eliminando los vértices de valores $\theta^{\prime x}$ y $\theta^{\prime} y$ de los subgrafos $S^{\prime}{ }_{1}, S^{\prime}{ }_{2}$ y $S^{\prime}{ }_{3}$ según las secuencias de Figs.5e,f y posteriormente eliminando los vértices de valor $\Delta^{\prime}$ según la secuencia de Fig.5g. Como resultado se obtienen los esquemas de Figs.5h,i que se utilizan para determinar de manera exacta y en función de 1/El los movimientos de la deformada de $\pi_{1}$ cuyos valores se han redondeado a partir del segundo decimal: se comienza calculando un desplazamiento parcial $\Delta^{z_{1}}$ por $\mathrm{F}_{\mathrm{G}}$ (Fig.5i). "Ascendiendo" hasta $A$ se obtiene $\Delta^{z} A 2$, que con $\Delta^{z} A 1$ producido por $F_{H}$ hacen el desplazamiento de $A, D$ y G. A continuación se determinan los desplazamientos parciales restantes descendiendo desde $A$ hasta $C$ pasando por $B$ y por el arco que une A con C. Desde estos tres vértices se "desciende" en Fig.5h hacia los vértices laterales: primero se obtienen los giros de $B$ y después los restantes. 
Finalmente el axil de la barra BE se ha obtenido considerando el extremo B y aplicando (12) en el subgrafo de Fig.5j que se ha extraído de Fig.5a y completado con el arco radial del pilar de extremo B. El resultado (16) es negativo y significa que la barra está traccionada según el criterio de Fig.1a.

$\mathrm{f}_{\mathrm{BE}}^{\mathrm{z}}=-\frac{9}{16} \Delta_{\mathrm{B}}^{\mathrm{z}}+\frac{3}{16} \Delta_{\mathrm{A}}^{\mathrm{z}}-\frac{3}{8} \theta_{\mathrm{A}}^{\mathrm{y}}+\frac{3}{8} \theta_{\mathrm{C}}^{\mathrm{y}}+\frac{3}{16} \Delta_{\mathrm{C}}^{\mathrm{z}}+\frac{3}{8} \theta_{\mathrm{B}}^{\mathrm{x}} \approx-0,44 \mathrm{Kn}$

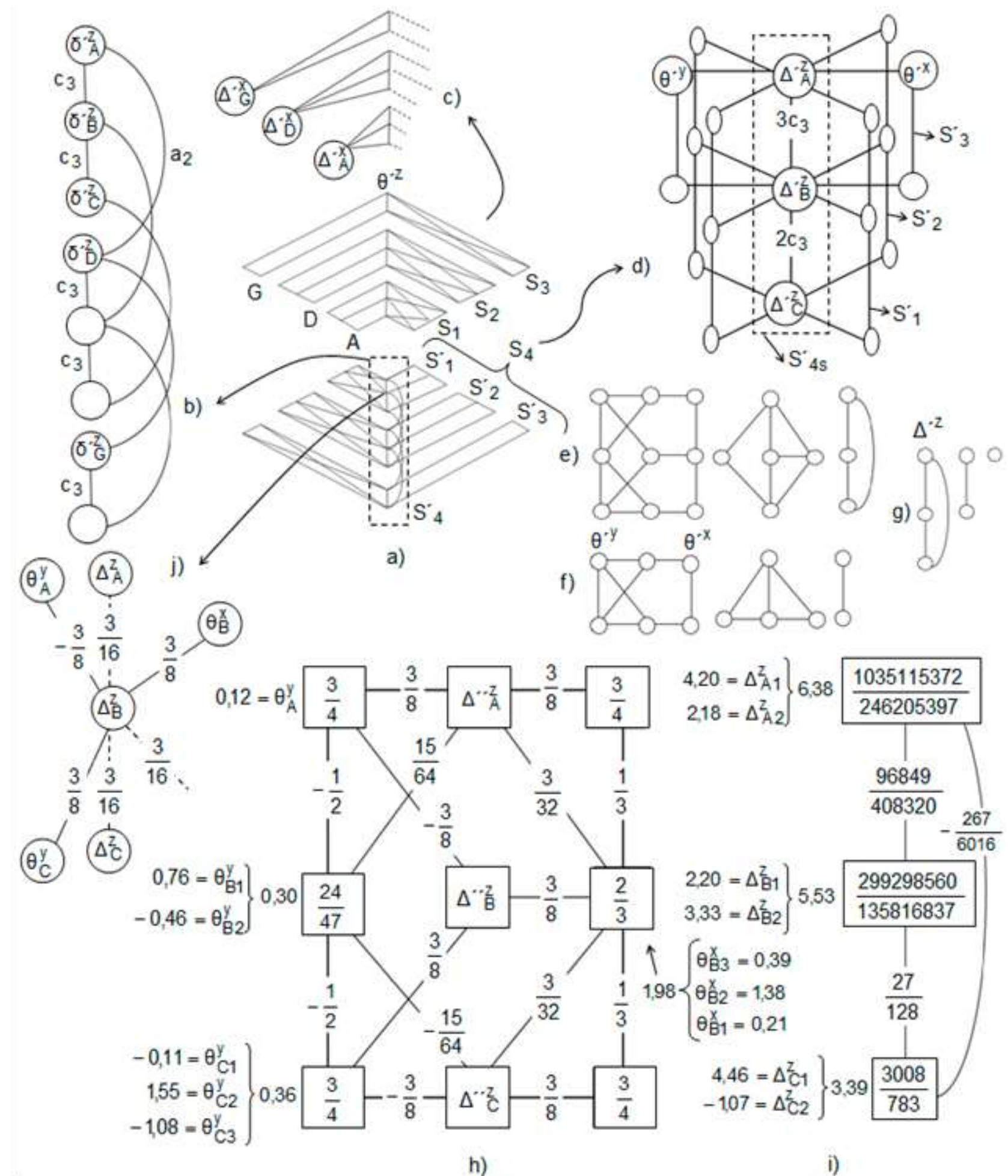

Fig.5: Análisis semigráfico del pórtico de Fig.3c. a) $G_{1}$ incompleto; b) Detalle; c) Simplificación de, $S_{1}, S_{2}$ y $S_{3}$; d) Simplificación de $S_{4}$; e), f) y g) Orden de eliminación de los vértices de $S^{\prime}{ }_{1}, S^{\prime}{ }_{3}$ y de los del tipo $\Delta^{\prime}$, respectivamente; h) Cálculo de giros i) Cálculo de desplazamientos, j) Subgrafo para calcular fź $\mathrm{BE}$ 


\section{CONCLUSIONES}

Es posible analizar semigráficamente de manera exacta en régimen elástico y en Teoría de Primer Orden ciertas estructuras hiperestáticas de edificación cuyas tipologías son habituales en obra nueva considerando el efecto de las redundantes y empleando grafos extraídos directamente de la geometría general de los modelos.

Es posible justificar cualitativamente las operaciones del método de Gauss que resuelve los sistemas de ecuaciones lineales procedentes de los análisis al poderlas relacionar con el comportamiento mecánico de los modelos.

\section{AGRADECIMIENTOS}

El autor agradece al Catedrático de Estructuras de Edificación de la Escuela de Arquitectura de Valencia (España), Dn. Agustín Pérez García, por sus recomendaciones previas a la elaboración de este trabajo.

\section{REFERENCIAS}

Beghini, L. y otros cuatro autores, Structural Optimization using Graphic Statics, doi: 10.1007/s00158-0131002-x, Struct Multidisc Optim (por suscripción), 49(3), 351-366 (2014)

Block, P. y L. Lachauer, Three-dimensional funicular analysis of masonry vaults, doi: 10.1016/j.mechrescom.2013.11.010, Mechanics Research Communications (por suscripción), 56, 53-60, (2014)

Calavera, J., Proyecto y cálculo de estructuras de hormigón, tomo 1, 2ª edición, 124-125, Intemac, Madrid, España (2008)

De la Fuente, J.L., Técnicas de cálculo para sistemas de ecuaciones, programación lineal y programación entera, 235, Reverté SA, Barcelona, España (1998)

Dobry, R., Simplified Methods in Soil Dinamics, doi:10.1016/j.soildyn.2014.02.008, Soil Dynamics and Earthquake Engineering (por suscripción), 61, 246-268, (2014)

Fivet, C. y D. Zastavni, Robert Maillart's key methods from the Salginatobel Bridge design process (1928), Journal of the International Association for Shell and Spatial Structures, ISSN: 1028-365X, 53(171), 39-47, (2012), (por suscripción), http://www.iass-structures.org/index.cfm/journal.home (2012)

Huanyun, W. y P. Chunhui, Teaching reform of Engineering Graphics on the relationship between hand drawing and computer drawing, International Conference of Information, Business and Education Technology, Beijing, China, 14 a 15 de Marzo, (2013)

Huerta, S., Structural Design in the Work of Gaudí, doi: 10.3763/asre.2006.4943, Architectural Science Review (por suscripción), 49(4), 324-339, (2006)

Kaveh, A. y B. Salimbahrami, Eigensolution of Symmetric Space Frames by Factorization of Their Graph Models, doi: 10.1260/136943309788251696, Advances in Structural Engineering (por suscripción), 12(2), 139-167 (2009)

Kaveh, A., H.A. Rahimi y L. Shahryari, Buckling Load of Symmetric Planar Frames with Semi-rigid Joints Using Graph Theory, International Journal of Civil Engineering, ISSN: 1735-0522 (en línea), 4(3), 157-175 (2006), http://ijce.iust.ac.ir/browse.php?mag_id=29\&slc_lang=en\&sid=1, Acceso: 1 de Septiembre (2006)

Keitel, H. y otros cuatro autores, Evaluation of coupled partial models in structural engineering using graph theory and sensitivity analysis, doi: 10.1016/j.engstruct.2011.08.009, Engineering Structures (por suscripción), 33(12), 3726-3736 (2011)

Lacort, A.G., Análisis manual aproximado y exacto de pórticos espaciales mediante cargas descompuestas, doi: 10.4067/S0718-07642011000200012, Inf. Tecnol (en línea), 22(2), 107-120 (2011)

Nilesh, T., S. Shubhada, Use of Graphical Technique for Stability Analysis of Embankment, IOSR Journal of Mechanical and Civil Engineering, ISSN: 2278-1684 (en linea). 3(5), 1-10 (2012), http://figshare.com/articles/Use_of_Graphical_Technique_for_Stability_Analysis_of_Embankment/1141399, Acceso: 16 de Agosto (2014) 
Parter, S., The use of graphs in Gauss Elimination, doi: 10.1137/1003021, SIAM rev (por suscripción), 3(2), $119-130(1961)$

Pissanetzky, S., Sparse Matrix Technology, 94-112, Academic Press Inc, Londres, UK (1984)

Sánchez-Beitia, S., Analysis of the collapse mechanisms in uncracked arches: the role of friction forces and stereotomy in masonry, doi: 10.1016/j.engfailanal.2013.02.021, Engineering Failure Analysis (por suscripción), 35(Part 1), 326-333 (2013)

Seung-Eok. K., P. Moon-Ho y C. Se-Hyu, Direct design of three dimensional frames using practical advanced analysis, doi: 10.1016/S0141-0296(01)00041-4,Engineering Structures (por suscripción), 23(11), 1491-1502 (2001)

Spielman, D.A., Algorithms, Graph Theory and Linear Equations in Laplacian Matrices, Proceedings of the International Congress of Mathematicians, Hyderabad, India, 19 a 27 de Agosto (2010)

Yanyan, S. y H. Hong, A Simplified Approach for Predicting Bridge Pier Responses Subjected to Barge Impact Loading, doi: 10.1260/1369-4332.17.1.11, Advances in Structural Engineering (por suscripción), 17(1), 11-24, (2014)

Van Mele, $\mathrm{T}$ y otros tres autores, Geometry-based understanding of structures, Journal of the International Association for Shell and Spatial Structures, ISSN: 1028-365X, 53(174), 285-295, (2012), (por suscripción), http://www.iass-structures.org/index.cfm/journal.home (2012) 